\title{
Signal recognition models compared for random and Markov
} \section{presentation sequences*}

\author{
ARTHUR SANDUSKY \\ University of California, Santa Barbara, California 93106
}

Models of two-category signal recognition are compared to data from a variety of experimental conditions. For recognition, one of two signals $\left(S_{1}, S_{2}\right)$ which vary slightly on some simple physical dimension is presented on each trial, and $O$ is to identify $\left(I_{1}, I_{2}\right)$ which signal was presented. In general, Os show a decrease in both $\operatorname{Pr}\left(\mathrm{I}_{2} / \mathrm{S}_{2}\right)$ and $\operatorname{Pr}\left(\mathrm{I}_{2} / \mathrm{S}_{1}\right)$ for either greater $\operatorname{Pr}\left(\mathrm{S}_{2}\right)$ or, sequentially, for greater $\mathrm{S}_{2}$ recency. These effects are described as probability and sequential contrast, respectively. The memory state model (MS) describes a three-state threshold process with responses determined by a simple first-order Markov process which depends on the sensory state and response on the immediately preceding trial. The memory trace regression model (MTR) assumes that 0 compares the observed signal event with the memory trace of the previous signal event. When the difference is large, the sign of the difference determines the response; when it is small, the response depends on the preceding response. The memory trace is assumed to regress toward the mean signal value. Both models accurately predict the observed bias changes as a function of signal probabilities and of the subsequence of events on the previous trial. The response axioms of the MTR model are modified to predict the results for individual Os for Markov chains of signal events. The response axioms of the MS model are modified to predict responding when information is given to $O$ concerning signal probabilities. Although both models do well under all conditions, the MS model uses fewer parameters and correctly predicts the direction of higher order sequential dependencies.

Two-category signal recognition is the simplest case of absolute category judgment. One of two possible signals $\left(S_{1}, S_{2}\right)$ is presented on each trial. Both signals are easily detected and differ on some simple physical dimension, but are difficult to discriminate from each other. The O's task is to identify $\left(I_{1}, I_{2}\right)$, on each trial, which of the two signals was presented.

Thurstone (1927) described the more general version of this type of experiment from which evolved methods of interval scaling of stimuli using category judgments (Torgerson, 1958). Since his interest was in scaling rather than in the details of the judgment process, he assumed that contextual effects defined by the presentation schedule were negligible. However, much research since then has focused largely on these effects as a means of studying the judgment process itself.

Stimulus presentation probabilities and sequential dependencies have been found to markedly influence judgmental responses. In the case of two-choice recognition, responses vary

*The author wishes to thank Allen Parducci and Morton Friedman of UCLA for their guidance in the completion of the author's doctoral dissertation, on which this report is, in part, based. Computing was performed with the assistance of the UCSB and UCLA computing facilities. inversely with signal probability (Parducci \& Sandusky, 1965; Tanner et al, 1967). When the signal presentation probabilities are varied, there is a tendency to identify both signals with the response appropriate to the less probable signal. Both $\operatorname{Pr}\left(\mathrm{I}_{2} / \mathrm{S}_{2}\right)$ and $\operatorname{Pr}\left(\mathrm{I}_{2} / \mathrm{S}_{1}\right)$ increase with decreases in $\operatorname{Pr}\left(\mathrm{S}_{2}\right)$, an effect found with many judgmental tasks and called probability contrast.

Responses show a similar contrast effect dependent on the subsequence of preceding stimuli. In particular, the presentation of an $S_{2}$ signal decreases the probability of an $I_{2}$ response to either signal on the following trial. It has been proposed that these sequential dependencies can account for probability contrast, since in an otherwise random sequence the more probable signal is more likely to precede either event. Averaging responses to each signal over preceding subsequences would then produce probability contrast whenever there is sequential contrast.

In the absence of informational feedback about the signal events, Os learn little about the signal probabilities; mean response probabilities vary directly, but slightly, with signal probabilities. When the response to the preceding signal is taken into account, a sizable response repetition tendency has been noted, but it has been shown that this cannot account for the sequential contrast (Parducci \& Sandusky, 1965).

\section{MEMORY STATE MODEL}

A number of theories of signal recognition for the case in which no feedback information is provided have been proposed to account for these results (Parducci, 1964; Parducci \& Sandusky, 1965; Sandusky, 1966; Tanner et al, 1967). Two of the major contenders will be compared here. The memory state model (MS) is a model previously proposed by Sandusky (Parducci \& Sandusky, 1965; Sandusky, 1966) as the band criterion model. This model is modified here to allow for alternative probabilistic response strategies. In its present form it is described as a three-state threshold model for sensory activation with the decision component a first-order Markov process in the sensory response states.

The probability of a true recognition for each of the two signals is assumed to be a constant, $\alpha$, and $\alpha_{2}$, as shown in Fig. 1. Responding is deterministic when the signal is recognized, and the appropriate identifying response is given. If neither signal is recognized, the response depends on the sensory state on the immediately preceding trial. If the signal was not recognized on the preceding trial, then the $O$ adopts a response repetition or alternation strategy and repeats the preceding response with probability $v$. If the last signal was recognized and the present signal is not, he assumes that the signal has shifted and modifies his response strategy in the direction of response alternation. In the presence of prior stimulus information he repeats the preceding response with probability $\rho$, where $\rho \leqslant v$.

These assumptions suggest that the perceptual frame of reference is unaffected by the signal probabilities and the sequential structure. What the $O$ does in the nonrecognition state is to use the sensory information on the preceding trial to modify his response strategy. Whenever the sensory state has shifted, he is more likely than not to shift his response. Whenever the sensory state remains the same, he is more likely to repeat the response.

These axioms describe the transition matrix of a first-order Markov process in four sensory response states: $\left(\psi_{1}\right) S_{2}$ is recognized and response $I_{2}$ is given, $\left(\psi_{2}\right) S_{1}$ is recognized and $I_{1}$ is given, $\left(\psi_{3}\right)$ the signal is not recognized and $I_{2}$ is given, $\left(\psi_{4}\right)$ the signal is not recognized and $I_{1}$ is given. The transition matrix for this process, given in Table 1 , describes the theoretical probability of being in State $\psi_{j}$ on Trial $n$, given that the process was in State $\psi_{i}$ on Trial $n-1$, 


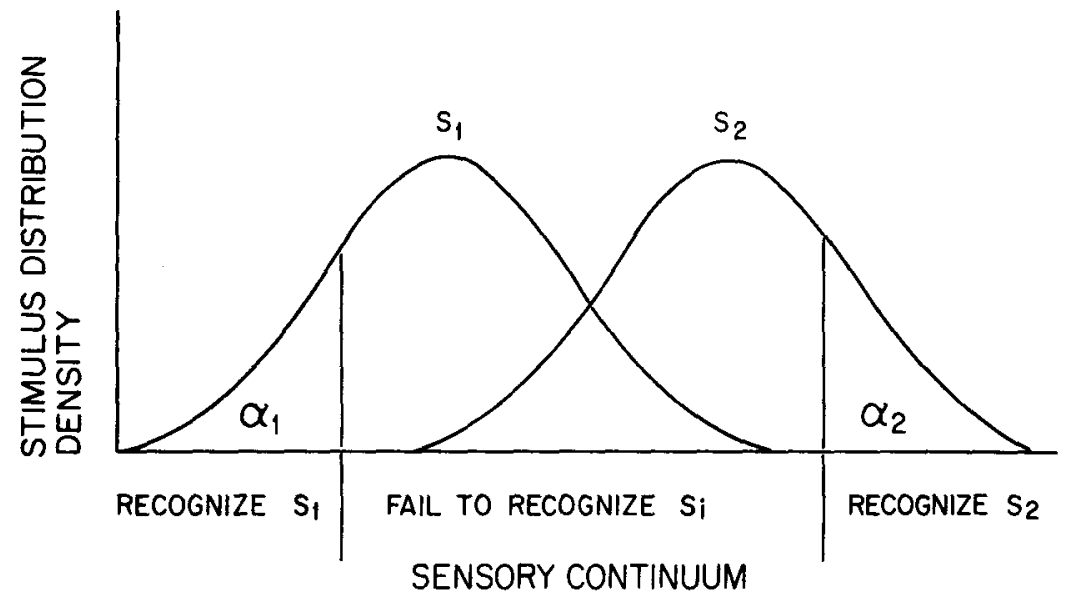

Fig. 1. Representation of the three sensory states for the memory state model.

where $\operatorname{Pr}\left(\mathrm{S}_{2}\right)=\gamma$ and $\alpha_{1}=\alpha_{2}=\alpha$. From this matrix, the asymptotic probabilities of each of the four states and the asymptotic mean probability of an $I_{2}$ response, given any sequence of signal and response events, can be derived.

\section{MEMORY TRACE \\ REGRESSION MODEL}

The memory state model is to be compared with the memory trace regression model (MTR) developed by Haller (1969) as the memory recognition model and is quite similar to a model previously developed from Helson's (1965) adaptation-level theory (Sandusky, 1966). The sensory activation process for each signal is assumed to be a continuous normal distribution of effects, with the $S_{1}$ mean equal to zero, the $S_{2}$ mean equal to one, and common variance. The signal effect on each trial $\left(s_{i}\right)$ is compared to the memory trace of the preceding signal effect $\left(t_{k}\right)$. If the difference, $d_{i k}=s_{i}-t_{k}$, is greater than $\delta_{1}$, then the $I_{2}$ response is given; if it is less than $\delta_{2}$, then $I_{1}$ is given where $\delta_{1} \geqslant \delta_{2}$. Otherwise, the preceding response is repeated. The memory trace of the signal effect regresses toward previously observed signal values as a linear average of the form:

$$
t_{n}=(1-\alpha) t_{n-1}+\alpha s_{i, n-1} .
$$

Table 1

The Transition Matrix for the Memory State Model of Recognition for Random Sequence of Signal Events. A prime on the parameter indicates that its value is subtracted from 1.0 .

\begin{tabular}{cccccc}
\hline & & \multicolumn{4}{c}{ State on Trial n } \\
\cline { 2 - 6 } & $\psi_{1}$ & $\psi_{2}$ & $\psi_{3}$ & $\psi_{4}$ \\
\hline State & $\psi_{1}$ & $\gamma \alpha$ & $\gamma^{\prime} \alpha$ & $\alpha^{\prime} \rho$ & $\alpha^{\prime} \rho^{\prime}$ \\
on & $\psi_{2}$ & $\gamma \alpha$ & $\gamma^{\prime} \alpha$ & $\alpha^{\prime} \rho$ & $\alpha^{\prime} \rho$ \\
Trial & $\psi_{3}$ & $\gamma \alpha$ & $\gamma^{\prime} \alpha$ & $\alpha^{\prime} v$ & $\alpha^{\prime} v^{\prime}$ \\
n-1 & $\psi_{4}$ & $\gamma \alpha$ & $\gamma^{\prime} \alpha$ & $\alpha^{\prime} v^{\prime}$ & $\alpha^{\prime} v$ \\
\hline
\end{tabular}

It is assumed that the signal effect on a trial is independent of the trace active on that trial and that the $d_{i k}$ are normally distributed with variance $\sigma_{D}{ }^{2}$. It should be noted that the $d_{i k}$ can only be approximately normal since the derived trace distribution is, in general, not normally distributed. The consequence of this assumption has not yet been investigated, but it is assumed here that the error introduced by this approximation is small.

For random sequences, the asymptotic mean trace value given $S_{2}$ on Trial $n-1$ is

$$
t_{2}=(1-\alpha) \gamma+\alpha
$$

and given $S_{1}$ on Trial $n-1$ is

$$
t_{1}=(1-\alpha) \gamma
$$

Assuming that the asymptotic trace values are normally distributed, approximate derivations can be made for any sequence of signal events. Derivations have been presented for subsequences of the type $\operatorname{Pr}\left(I_{2, n} / S_{k, n} I_{j, n-1} S_{i, n-1}\right)$ for random sequences of signal events (Tanner et al, 1967). These derivations use the additional approximation that the expected value of $\left(t_{n} / S_{i, n-1}\right)=$ $\left(t_{n} / I_{j, n}-1 S_{i, n-1}\right)$.

$T h$ is model assumes that the perceptual frame of reference is established by comparison of current stimuli with the $O$ 's memory of the preceding event. This memory is distorted by previous events-it is a running average over all previous events, with greater weighting for recency. The memory distortion then is strongly affected by signal probabilities and sequential structure. In addition, the sensory effects of the signals and, as a consequence, the memory effect are both assumed to be subject to random fluctuations comparable to those described by
Thurstone (1927). The comparison of the signal effect to the memory gives rise to a continuum of possible differences, the distribution of these being determined by the preceding stimulus sequence. The response is determined by the sign of the difference; large differences give rise to unequivocal responses, small differences are treated as no differences and are discarded in favor of response preservation.

One model (MS) assumes an invariance of discrete sensory effects; contextual variations in responses are ascribed solely to guessing strategies. The other (MTR) assumes that contextual variations in responding are ascribed directly to a continuum of sensory memory variations with guesses playing only a trivial role. It is remarkable that two theories with such divergent bases can so closely parallel each other in terms of predicted response probabilities, as will be shown in empirical comparisons made in this paper.

COMPARISON TO DATA WITH SIGNAL PROBABILITIES VARIED

Both models have been shown to be reasonably successful in accounting for the first-order sequential statistics as a function of presentation probabilities for group data. The data for which the present comparisons are made is taken from a previously published experiment by Parducci and Sandusky (1965). The Os were to recognize which of two lights differing in horizontal position (left or right) was flashed on each trial. The room was completely dark except for a fixation light which was on constantly and centered 11 in. above the $1 / 2$-in.-separated stimulus lights which were $5 \mathrm{ft}$ from $O$. Two signal probability distributions, $\operatorname{Pr}\left(\mathrm{S}_{2}\right)=.2$ or $\operatorname{Pr}\left(S_{2}\right)=.8$, were presented to two independent groups of 40 Ss. The details of the experimental method may be found in the earlier paper.

The effect of signal probability on mean response probabilities to the two signals is shown in Fig. 2-A. The ROC plot shows the probability of a "right" response to a right stimulus, $\operatorname{Pr}\left(\mathrm{I}_{2} / \mathrm{S}_{2}\right)$, against the probability of a "right" response to a left stimulus, $\operatorname{Pr}\left(I_{2} / S_{1}\right)$. The point for $\operatorname{Pr}\left(S_{2}\right)=.2$ is above and to the right of the point for $\operatorname{Pr}\left(S_{2}\right)=.8$, showing a contrast bias shift away from the more probable signal.

The ROC plot of the first-order stimulus sequential effects is shown in Fig. 2-B. For both probability schedules, the point for $S_{1}$ on Trial $n-1$ is up and to the right of that for $S_{2}$ on $n-1$, indicating a contrast bias shift away from the most recent preceding signal. The differential averaging of these points as 


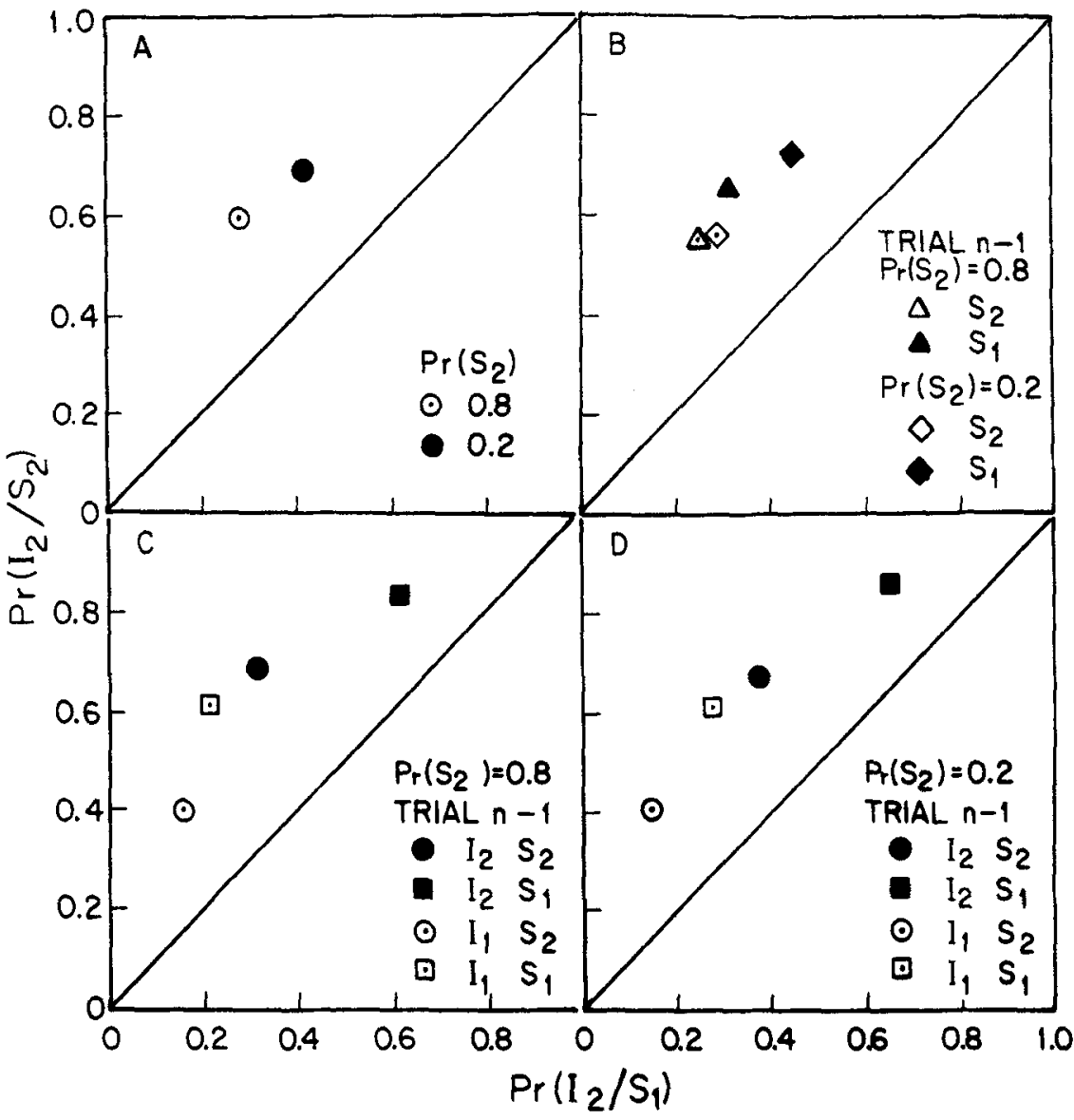

Fig. 2. ROC plot of the results of the Parducci and Sandusky (1965) recognition experiment for signal probability effects. Statistics plotted are given in the legend.

a consequence of the different signal probabilities results in the probability contrast shown in Fig. 1-A.

The data for theoretical comparison of the signal probability effect was tabulated and is shown in Fig. 2-D and $-C$. This graph plots $\operatorname{Pr}\left(I_{2, n} / S_{2, n} I_{j, n-1} S_{i, n-1}\right)$ against $\operatorname{Pr}\left(I_{2, n} / S_{1, n}, I_{i, n-1} S_{i, n-1}\right)$, where $i$ and $j$ take on the values 1 and 2 as indicated in the legend. In addition to the signal contrast effect (circles vs squares), these data take the preceding response into account and show a strong response assimilation effect (filled vs open symbols). Responses following an $I_{2}$ response are above and to the right of those following an $I_{1}$ response, indicating a strong tendency to repeat responses. However, it is clear from the graph that this repetition tendency alone cannot account for the stimulus contrast effect since stimulus effects are still present.

\section{THEORETICAL COMPARISONS}

The parameters for both the MS and MTR models were estimated so as to minimize the chi-square function for the difference between the predicted an $F$ ratio to allow comparison between the accuracy of the models (see Atkinson et al, 1965, p. 395). The $F$ ratio is not significant.

The fits of both models are reasonably good, considering that the data are pooled over groups of Os. Neither theory is such that this type of averaging is strictly appropriate. The average of the predictions for individual $O s$ is not expected to be the predictions for the data averaged over Os. What is needed for a more careful comparison is a relatively large amount of data to be collected from a relatively small number of Os with predictions made for individual Os' data.

Further, these data showed no sequential effects of order higher than the first-order effects. Responses were found to be independent of the signal on Trial $n-2$. However, both theories predict some effect of the events on Trial $n-2$. Again, what is needed are data for individual Os with enough data to examine these higher order effects.

Finally, there is some question concerning the effects of sequence apart from the overall values of the signal probabilities. For these reasons, it was decided to use a relatively small number of Os, many trials, and Markov sequences of signals with $\operatorname{Pr}\left(S_{2}\right)=.5$. In this way, the sequence can be manipulated without affecting signal probabilities.

\section{MARKOV SEQUENCES EXPERIMENT}

Method

The same two-choice position recognition task was used as in the previous experiment. On each trial, one of two lights, differing in horizontal position, was flashed and (see Suppes \& Atkinson, 1960, p. 58). The estimates were obtained by using a high-speed computer to calculate the chi-square function over a grid of possible values of the parameters and selecting the values that approximated a minimum. Since the data for this task proved relatively symmetric for the two responses, neither model was fitted to include position effects of the signals. Thus, it was assumed that $\alpha_{1}=\alpha_{2}$ for the MS model and $\delta_{1}=-\delta_{2}$ for the MTR model.

The predicted proportions for the two models, the observed proportions (Obs), and the number of observations for each proportion (N) are presented in Table 2. The parameters for the MS model are $\alpha=.28, v=.82$, and $\rho=.26$; and for the MTR model are $\alpha=.73$, $\delta=.49$, and $\sigma_{\mathrm{D}}=1.24$. The chi-square deviations divided by the associated degrees of freedom are $\mathbf{1 2 . 8 3}$ and $\mathbf{9 . 4 2}$ for the MS and MTR models, respectively. The ratio of the chi squares for the two different models can be roughly interpreted as
Table 2

Observed and Predicted Values of $\operatorname{Pr}\left(I_{2, n} / S_{k, n I}, n-1 S_{i, n-1}\right)$ for the Parducci and Sandusky (1965) Experiment

\begin{tabular}{|c|c|c|c|c|c|}
\hline \multirow[b]{2}{*}{$\operatorname{Pr}\left(S_{2}\right)$} & \multirow[b]{2}{*}{ Seq. } & \multicolumn{2}{|c|}{ Predicted } & \multirow[b]{2}{*}{ Obs. } & \multirow[b]{2}{*}{$\mathbf{N}$} \\
\hline & & MS & MTR & & \\
\hline .80 & $\begin{array}{l}S_{2} I_{2} S_{2} \\
S_{1} I_{2} S_{2} \\
S_{2} I_{2} S_{1} \\
S_{1} I_{2} S_{1} \\
S_{2} I_{2} S_{2} \\
S_{1} I_{1} S_{2} \\
S_{2} I_{1} S_{1} \\
S_{1} I_{1} S_{1}\end{array}$ & $\begin{array}{l}.68 \\
.39 \\
.87 \\
.59 \\
.41 \\
.13 \\
.58 \\
.29\end{array}$ & $\begin{array}{l}.67 \\
.36 \\
.85 \\
.59 \\
.36 \\
.12 \\
.59 \\
.28\end{array}$ & $\begin{array}{l}.68 \\
.32 \\
.83 \\
.62 \\
.40 \\
.16 \\
.62 \\
.22\end{array}$ & $\begin{array}{r}4464 \\
1105 \\
480 \\
134 \\
3143 \\
813 \\
1428 \\
323\end{array}$ \\
\hline .20 & $\begin{array}{l}S_{2} I_{2} S_{2} \\
S_{1} I_{2} S_{2} \\
S_{2} I_{2} S_{1} \\
S_{1} I_{2} S_{1} \\
S_{2} I_{1} S_{2} \\
S_{1} I_{1} S_{2} \\
S_{2} I_{1} S_{1} \\
S_{1} I_{1} S_{1}\end{array}$ & $\begin{array}{l}.71 \\
.42 \\
.87 \\
.59 \\
.41 \\
.13 \\
.61 \\
.32\end{array}$ & $\begin{array}{l}.72 \\
.41 \\
.87 \\
.64 \\
.41 \\
.15 \\
.64 \\
.33\end{array}$ & $\begin{array}{l}.67 \\
.37 \\
.86 \\
.65 \\
.40 \\
.14 \\
.62 \\
.29\end{array}$ & $\begin{array}{r}312 \\
1337 \\
789 \\
3115 \\
145 \\
601 \\
2035 \\
4492\end{array}$ \\
\hline$x^{2} / d f$ & (dev.) & 11.84 & 8.63 & & \\
\hline
\end{tabular}




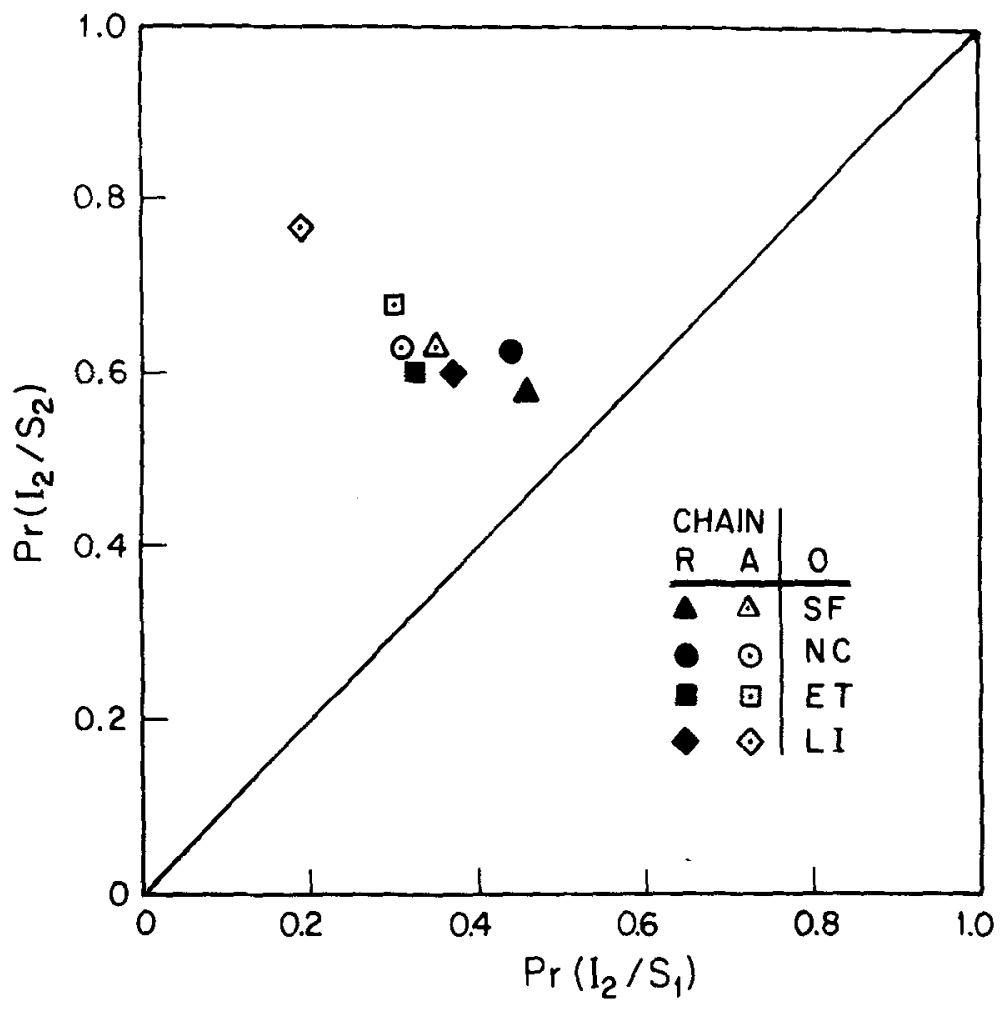

Fig. 3. ROC plot of $\operatorname{Pr}\left(\mathrm{I}_{2} / \mathrm{S}_{2}\right)$ against $\operatorname{Pr}\left(\mathrm{I}_{2} / \mathrm{S}_{1}\right)$ for the four $\mathrm{Os}$ in the Markov sequences experiment. $\operatorname{Pr}\left(S_{n}=S_{n+1}\right)=.8$ for R-chains and .2 for A-chains.

$O$ 's task was to indicate in which position the flash occurred.

\section{Observers}

The Os were four paid undergraduate students in psychology. They were required to perform at a better-than-chance level of accuracy on two presentations of random sequences before being selected. Only one $O$ was rejected by this criterion. The four Os selected will be referred to by their initials; S.F. was male, and L.I., E.T., and N.C. were females.

\section{Conditions}

The two types of Markov chains used for the sequences of signal events can be characterized by the probability of a signal repetition, $\mu=\operatorname{Pr}\left(S_{i, n+1}=S_{i, n}\right)$ with $\operatorname{Pr}\left(S_{2}\right)=.5$. For chains with high signal repetition rates, $R$-chains, $\mu=.8$. For chains with high signal alternation rates, A-chains, $\mu=.2$. One hundred chains of length 320 for both values of $\mu$, random except for the above restrictions, were generated by a computer program and punched on paper tape for stimulus programming.

\section{Procedure}

Before any responses were recorded, the four Os were each presented with five $A$ and five $R$ practice chains of length 320. For the experimental trials, two series of five $A$ - and five $R$ - two preceding signal events, are approximately independent of preceding events. The number of parameters used for each model is subtracted from the degrees of freedom in these evaluations, a course which Suppes and Atkinson (1960) assert is conservative.

The difference in response probabilities between the first and second half of each session of 300 trials for the eight first-order signal and response dependencies of the type $\operatorname{Pr}\left(I_{2, n} / S_{k, n} I_{j, n-1} S_{i, n-2}\right)$ were assessed by a chi-square test of stationarity. The sums of the chi squares were not significant for any of the Os.

A similar chi-square test was made for stationarity over four quarters of the experiment for the two chain types, and are significant for all four Os $(p<.01), x^{2}(48 \mathrm{df})=9.64,5.35$, 2.01, and 3.96 for S.F., L.I., E.T., and N.C., respectively. Eliminating the first quarter of S.F.'s data reduced the stationarity $x^{2}(32 \mathrm{df})$ to 4.39 . Consequently, the analysis of his data is based on the last three-quarters of the experiment. There appear to be many sources for the nonstationarity; however, no single source appears to be very large. Further, these chi squares are based on extremely large sample sizes and are presented here only for comparison with the order effects and the deviations from theoretical predictions.

Zero-order stimulus effects. The ROC plot of $\operatorname{Pr}\left(\mathrm{I}_{2} / \mathrm{S}_{2}\right)$ against $\operatorname{Pr}\left(\mathrm{I}_{2} / \mathrm{S}_{1}\right)$ for each $\mathrm{O}$ under the two Markov chain conditions is shown in Fig. 3. For all Os, the A-chain data appears to show greater sensitivity to the signals than does the R-chain data (open vs filled symbols).

It must be emphasized that the signals used with A-chains are the same as those used with R-chains, only the presentation sequence has been varied. The effect of this variation is to produce an apparent substantial increase in sensitivity to the signal differences with A-chains. This increase is expected for both models since they predict greater accuracy for signal alternation trials than for signal repetition trials.

For the MS model, this advantage on alternation trials is attributed to the response alternation strategy adopted on nonrecognition trials following trials on which the signal is recognized. On signal alternation trials, response alternations produce greater accuracy; on signal repetition trials, response alternation leads to less accuracy. For the MTR model, the advantage on alternation trials arises from the greater sensory differences between the observed stimulus and the memory trace, as compared to 


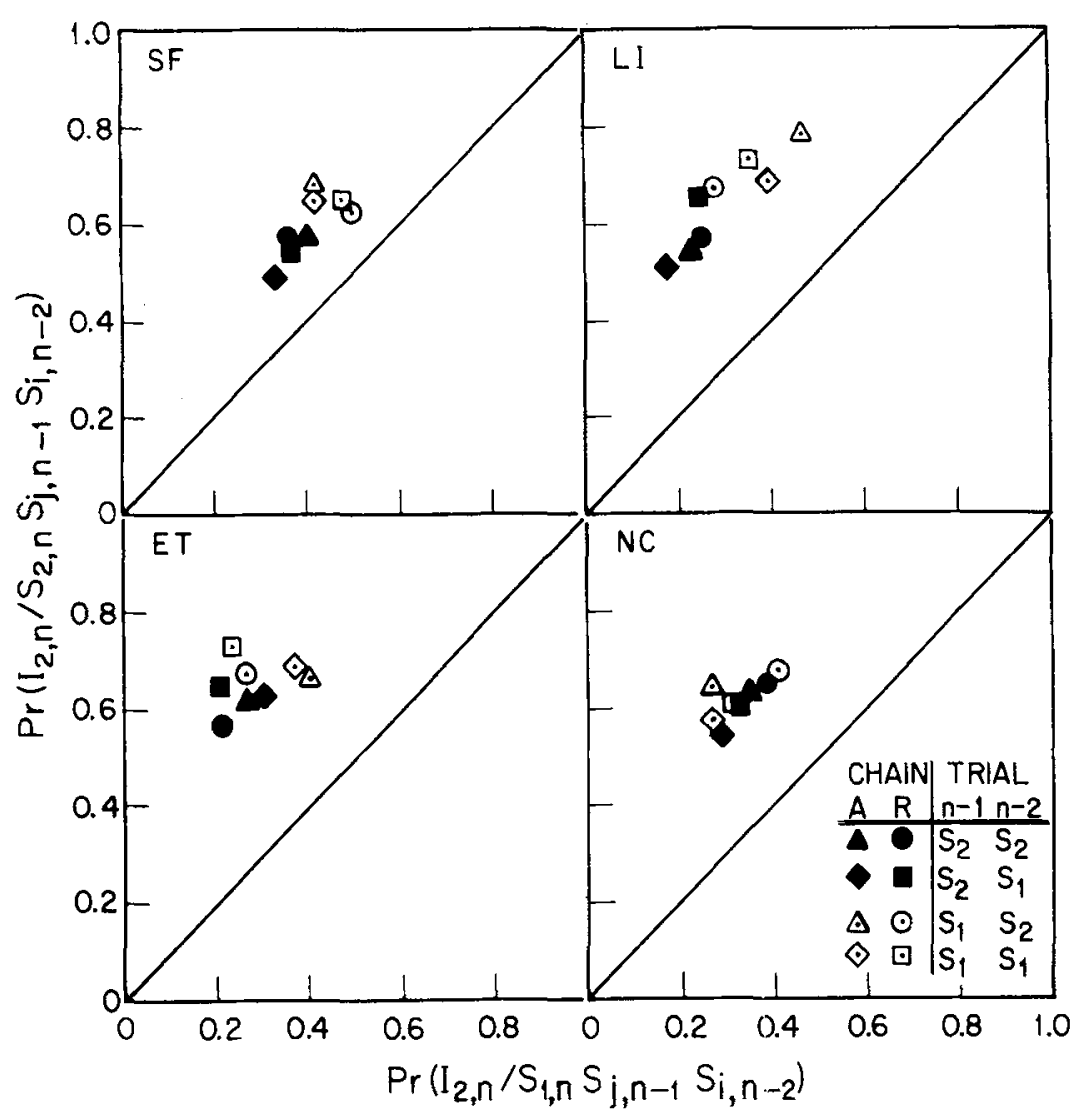

Fig. 4. ROC plot of the first- and second-order sequential signal effects for the four Os in the Markov sequences experiment.

differences observed on signal repetition trials.

Since A-chains have four times more signal alternations than do R-chains, the differential averaging would be expected to elevate mean accuracy for A-chains.

First and second-order stimulus effects. It can be seen from Fig. 4 that the apparent sensitivity differences attributable to chain type disappear when the previous subsequence of signal events on Trials $n-1$ and $n-2$ are taken into account. This graph plots $\operatorname{Pr}\left(I_{2, n} / S_{2, n} S_{j, n-1} S_{i, n-2}\right)$ against $\operatorname{Pr}\left(\mathrm{I}_{2, n} / \mathrm{S}_{1, n} \mathrm{~S}_{\mathrm{j}, \mathrm{n}-1} \mathrm{~S}_{\mathrm{i}, \mathrm{n}-2}\right)$. The chi squares for the first-order signal events which compare responses on signal alternation trials to signal repetition trials were significant for all Os $(p<.01)$ except N.C. and are $x^{2}(2 \mathrm{df})=68.31,31.63$, and 283.08 for S.F., L.I., and E.T., respectively. The direction of the effect shows a definite sequential bias contrast since the points for trials preceded by an $S_{1}$ signal are above and to the right of the respective points for trials preceded by an $S_{2}$ signal (open vs filled symbols).

The second-order signal effects which take into account the signal event on Trial $n-2$ are smaller but significant for all Os $(\mathrm{p}<.01)$, $\chi^{2}(4 \mathrm{df})=4.76,9.94,6.54$, and 6.01 effects. Again, the data for direct quantitative comparison of the theoretical models are response proportions of the type $\operatorname{Pr}\left(I_{2, n} / S_{k, n} I_{j, n-1} S_{i, n-1}\right)$. The ROC plot of $\operatorname{Pr}\left(I_{2, n} / S_{2, n} I_{j, n-1} S_{i, n-1}\right)$ against $\operatorname{Pr}\left(\mathrm{I}_{2, \mathrm{n}} / \mathrm{S}_{1, \mathrm{n}} \mathrm{I}_{\mathrm{j}, \mathrm{n}-1} \mathrm{~S}_{\mathrm{i}, \mathrm{n}-\mathrm{1}}\right)$ are shown for R-chains in Fig. 6 and for $A$ chains in Fig. 7 for each $O$. All four Os show a clear sequential signal contrast effect since the points for trials preceded by $S_{1}$ are above and to the right of the respective points for trials preceded by $\mathrm{S}_{2}$ (squares vs circles). However, the response effects differ for individual Os. For E.T. and N.C., there is a response assimilation effect, since the points for trials following $I_{1}$ are below and to the left of those following $\mathrm{I}_{2}$ (open vs filled symbols), showing a tendency to repeat successive responses. For S.F. and L.I., there is a definite response contrast effect since the points for trials following $I_{1}$ are above and to the right of those following $I_{2}$ (open vs filled symbols). This shows a tendency not to repeat, but to alternate successive responses.

\section{COMPARISONS TO DATA \\ FROM MARKOV CHAINS}

\section{Markov Derivations}

The derivations for Markov sequences of signals are straightforward for the MS model and involve no difficulties.

The derivations for the MTR model require finding the asymptotic values of $t_{1}$ and $t_{2}$ for the case of Markov sequences. The asymptotic value of $t$ for the linear operator defined in Eq. 1 is unchanged from that for random sequences. Derivations of this type have been presented elsewhere (Bush \& Mosteller, 1955 , p. 73). However, the values for $t_{1}$ and $t_{2}$ are changed

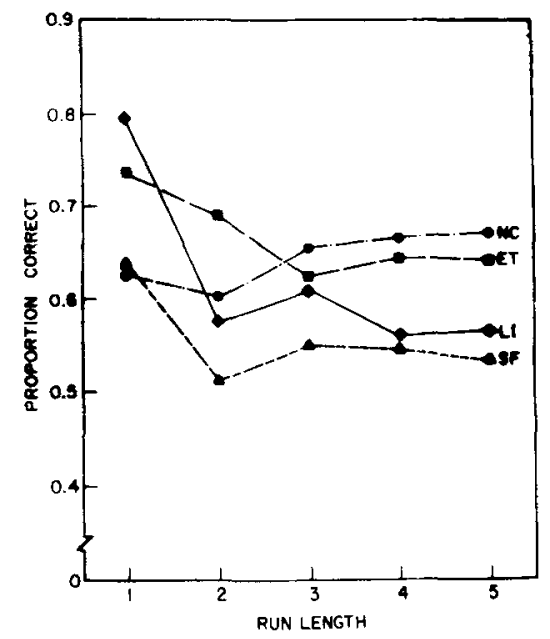

Fig. 5. Recognition accuracy as a function of the length of signal repetition runs averaged over the two signals for $\mathbf{R}$ chains. 


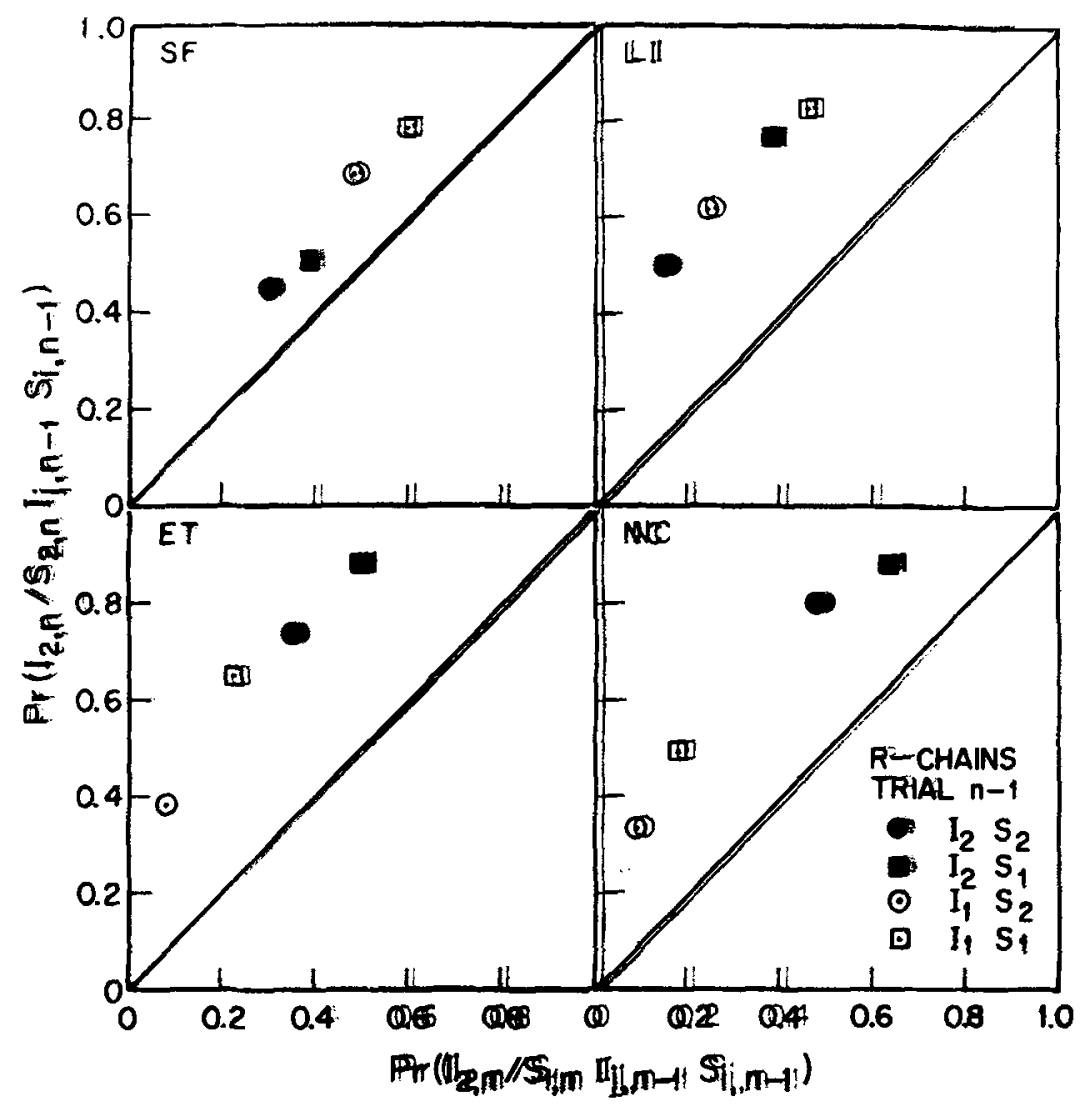

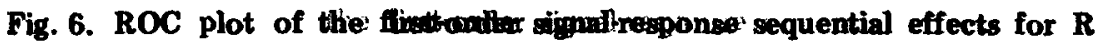

S.F. and L.I. also pronts porblems for the MTR modtel. Whien thee difference between the trucre and thee

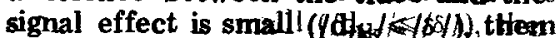
the $O$ is presumed to idtontffy the stimulus as not differentt from thee preceding identificatiomandiherequats his response. These two OS dio not repeat but, rather; adtornate suoth responses, which mades noo seowe in terms of the assumptions off thee model. In the recogriftionexpriment by Tanner et al (1967), OBs1i1 andili22 also show this response patterm.

The MTR model is therefonee modified in the direction aff previously presented adaptationalued model (Sandusky, 1966). Ratherthian assuming that the trace representesthee remembered value of the signilonitbe previous trial, it is here assumedlthint the trace is a remembered valuer of ftile neutral point on the contimumm between the two signals, consistent with Helson's (1965) notions of adaptation level. In this case; if $\left|\mathrm{d}_{\mathrm{ik}}\right| \leqslant|\delta|$, then the $\mathrm{O}$ adoptsis response strategy either repeating onr alternating responses. This involves the addition of a parameter, $\Delta$, which 1 characterizes the strategy and has value 1 if responses are repeated and -1 if they are alternated. The parameter is simply multiplied by $\delta_{j}$ in: the derivations given by Tanner et al (1967, Eq. 10). chains.

since the sequences of signal evemts ane not independent but are Mrollow chains. The equations for Mroctow: sequences of equiprobable events ane given by:

$$
t_{2}=\frac{(1-\alpha)(1-\mu)+\alpha}{2(1-\alpha)(1-\mu)+\alpha}
$$

and

$$
t_{1}=\frac{(1-\alpha)(1-\mu)}{2(1-\alpha)(1-\mu)+\alpha}
$$

where $(1-\mu)=\operatorname{Pr}\left(S_{j, n+1} \neq S_{i, n}\right)$. Given these equations, the derivations for the MTR model are straightforward.

\section{QUALITATIVE COMPARISONS}

The absence of continuing higher order contrast presents some difficulty for the MTR model. With continued repetitions of $S_{2}$, for example, the value of $t$ would come closer and closer to the mean value of the $S_{2}$ distribution. $D_{2_{2}}$ would have mean zero, and the response probabilities would be at a chance level. It is clear from the repetition run data in Fig. 5 that the response probabilities asymptote above chance. This is just what is expected from the MS model.

The response contrast noted for

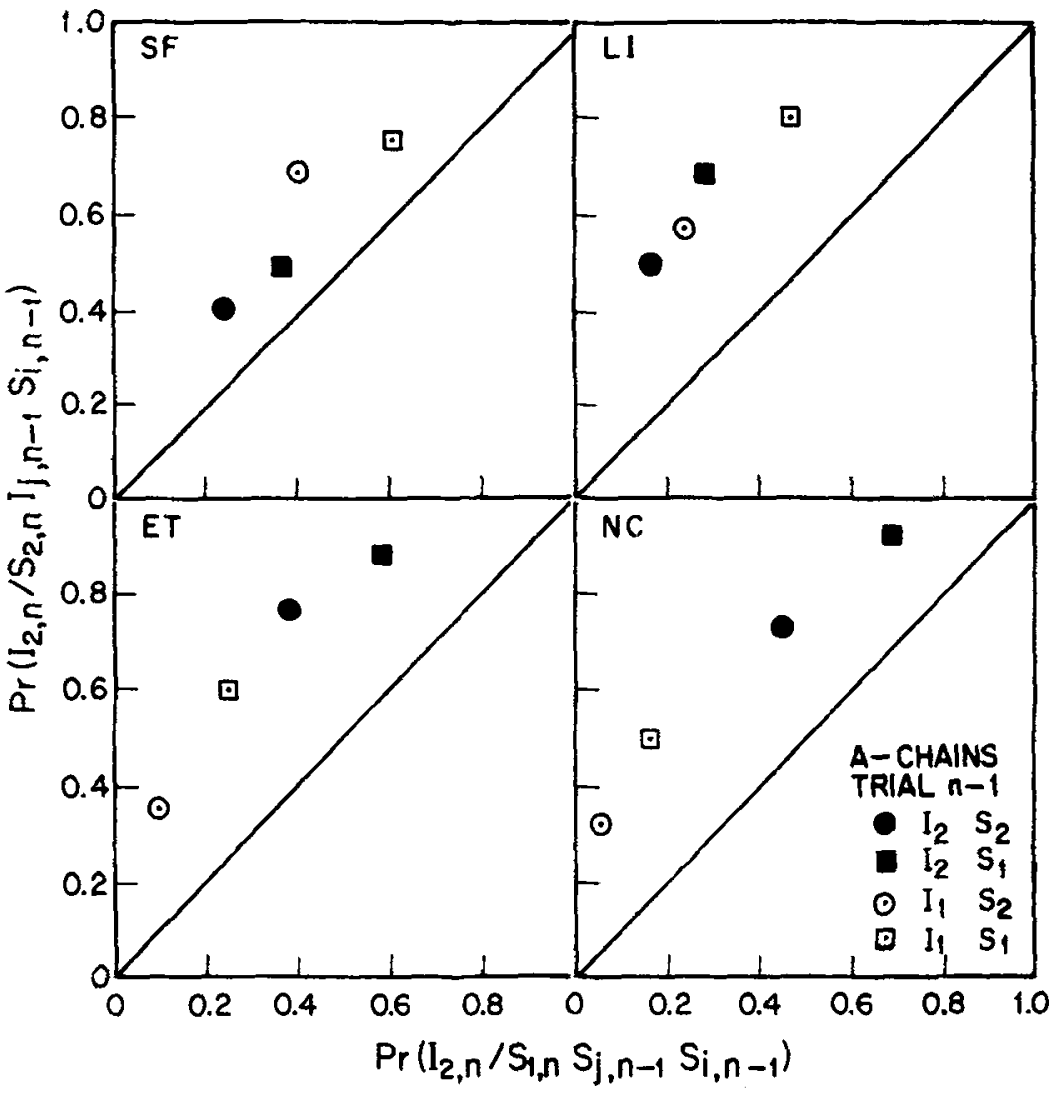

Fig. 7. ROC plot of the first-order signal-response sequential effects for $A$ chains. 
Table 3

stimated $P$ arameters for the Memory State lodel for the Four Os in the Markov Chain Experiment

\begin{tabular}{llcccc} 
& & \multicolumn{4}{c}{ Observer } \\
\cline { 3 - 6 } 1 odel & $\begin{array}{c}\text { Param- } \\
\text { eter }\end{array}$ & SF & LI & ET & NC \\
\hline \multirow{4}{*}{ MS } & $\alpha$ & .18 & .36 & .34 & .30 \\
& $v$ & .41 & .59 & .86 & .90 \\
& $\rho$ & .04 & .00 & .36 & .48 \\
\multirow{4}{*}{ MTR } & $\alpha$ & .56 & .46 & .52 & .54 \\
& $\sigma_{D}$ & 1.94 & .96 & .95 & .96 \\
& $\delta$ & .60 & .13 & .45 & .65 \\
& $\Delta$ & -1 & -1 & 1 & 1 \\
\hline
\end{tabular}

\section{QUANTITATIVE COMPARISONS}

The parameters for both models were estimated for each 0 for both chain types simultaneously, as described above, and are given in Table 3. The reduction in the response repetition tendency, given stimulus information on the previous trial $(v-\rho)$ for the MS model, was above .5 for all Os. As expected from the qualitative comparisons of the data, the value of $\Delta$ for the MTR model was -1 for S.F. and L.I. and +1 for E.T. and N.C.

The predicted values for the quantities $\operatorname{Pr}\left(I_{2, n} / S_{k, n} I_{j, n-1} S_{i, n-1}\right)$ for each $\mathrm{O}$ are given in Table 4 along with the observed proportions (Obs) and the number of observations (N). The fits for both models are extremely good. The average absolute value of the deviations from observed proportions is only .025 for the MS model and .030 for the MTR model. The chi-square deviations are given in the last row of the table. They are generally significant $(p<.01)$, except for S.F. under the MS model. However, these values tend to be about the same order of magnitude as the chi squares for stationarity, and such nonstationarity leads to decreased predictability. In no case does the $F$ ratio of the comparative fits show either model to be superior for these data.

\section{EXTENSION OF MODELS TO FEEDBACK}

Recently, the MTR model has been extended to the case for which Os are given knowledge of the signal probabilities and trial-by-trial feedback after each response as to which signal had been presented (Tanner et al, 1970). The results for recognition experiments with feedback are that Os tend to match response probabilities to the signal probabilities and they show both probability and sequential assimilation. The greater $\operatorname{Pr}\left(\mathrm{S}_{2}\right)$, the more probable is an $I_{2}$ identifying response to both $\mathrm{S}_{1}$ and $\mathrm{S}_{2}$. Sequentially, an $\mathrm{S}_{2}$ on Trial $\mathrm{n}-1$ increases the probability of an $\mathrm{I}_{2}$ response to either signal on Trial $n$. These are the effects typically found in signal detection experiments (Friedman et al, 1968; Atkinson et al, 1962).

To account for these dramatic effects of informational feedback with the MTR model, it was assumed that $O$ partially uses the feedback information to determine responses. The assumption is that when $d_{i k}$ (the difference between the observed and remembered value) falls between the limits $\delta_{1}$ and $\delta_{2}, O$ has two response tendencies: (1) to repeat the previous response and (2) to repeat the response designated as the previously correct response. Response probabilities are then a weighted average of these two tendencies, where $\omega$ is the weighting parameter (Tanner et al, 1970, Eq. 10). Further, it is assumed that $O$ varies the limits, $\delta_{1}$ and $\delta_{2}$, depending on the value of $\gamma$.

In order for the MS model to account for recognition results under both feedback and nonfeedback conditions, the rules for responding when neither stimulus is recognized must be restated. For the feedback case, the assumption is that when neither stimulus is recognized, responses tend to be given with the subjective estimate of the corresponding signal probabilities, where $\Gamma$ corresponds to the subjective probability of $\mathrm{S}_{2}$. Since previous responses are highly influential in these experiments, it is assumed that these response rates are additively modified by the previous state. When neither signal is recognized and the previous signal was recognized, the response rates are modified by the parameter $\epsilon$; when the previous signal was not recognized, these rates are modified by the parameter $\iota$.

The transition matrix for this statement of the model is given in Table 5.

For the case of no informational feedback, the assumption is that $\Gamma=.5$, and; in this instance, the transition matrix reduces to that given for random sequences in Table 1, where $\Gamma+\epsilon=\rho$ and $\Gamma+\imath=v$.

In the case of correct information, as given in the feedback conditon of Tanner et al (1970), the normative assumption is that $\Gamma=\gamma=\operatorname{Pr}\left(\mathrm{S}_{2}\right)$.

For a two-category tone recognition experiment in which feedback was given, Tanner et al (1970) require eight parameters to fit the data for two presentation probability schedules, $\operatorname{Pr}\left(\mathrm{S}_{2}\right)=.2$ or .8 , with the MTR model $\left(\omega=.73, \sigma_{\mathrm{D}}=.51\right.$,

Table 4

Observed and Predicted Values of $\operatorname{Pr}\left(I_{2, n} / S_{k, n} I_{j, n}-1 S_{i, n-1}\right)$ for the Markov Chain Experiment

\begin{tabular}{|c|c|c|c|c|c|c|c|c|c|c|c|c|c|c|c|c|c|}
\hline \multirow{3}{*}{$\begin{array}{c}\text { Markov } \\
\text { Chain }\end{array}$} & \multirow[b]{3}{*}{ Seq. } & \multicolumn{4}{|c|}{ O SF } & \multicolumn{4}{|c|}{ O LI } & \multicolumn{4}{|c|}{ O ET } & \multicolumn{4}{|c|}{ ONC } \\
\hline & & \multicolumn{2}{|c|}{ Predicted } & \multirow[b]{2}{*}{ Obs. } & \multirow[b]{2}{*}{$\mathbf{N}$} & \multicolumn{2}{|c|}{ Predicted } & \multirow[b]{2}{*}{ Obs. } & \multirow[b]{2}{*}{$\mathbf{N}$} & \multicolumn{2}{|c|}{ Predicted } & \multirow[b]{2}{*}{ Obs. } & \multirow[b]{2}{*}{$\mathbf{N}$} & \multicolumn{2}{|c|}{ Predicted } & \multirow[b]{2}{*}{ Obs. } & \multirow[b]{2}{*}{$\mathbf{N}$} \\
\hline & & MS & MTR & & & MS & MTR & & & MS & MTR & & & MS & MTR & & \\
\hline $\mathbf{R}$ & $\begin{array}{l}S_{2} I_{2} S_{2} \\
S_{1} I_{2} S_{2} \\
S_{2} I_{2} S_{1} \\
S_{1} I_{2} S_{1}\end{array}$ & $\begin{array}{l}.42 \\
.24 \\
.52 \\
.34\end{array}$ & $\begin{array}{l}.41 \\
.23 \\
.55 \\
.35\end{array}$ & $\begin{array}{l}.45 \\
.30 \\
.49 \\
.36\end{array}$ & $\begin{array}{l}952 \\
288 \\
223 \\
847\end{array}$ & $\begin{array}{l}.52 \\
.16 \\
.74 \\
.38\end{array}$ & $\begin{array}{l}.50 \\
.15 \\
.78 \\
.40\end{array}$ & $\begin{array}{l}.51 \\
.14 \\
.77 \\
.38\end{array}$ & $\begin{array}{r}1431 \\
402 \\
222 \\
864\end{array}$ & $\begin{array}{l}.73 \\
.40 \\
.90 \\
.57\end{array}$ & $\begin{array}{l}.74 \\
.34 \\
.91 \\
.62\end{array}$ & $\begin{array}{l}.73 \\
.34 \\
.87 \\
.59\end{array}$ & $\begin{array}{r}1473 \\
398 \\
206 \\
717\end{array}$ & $\begin{array}{l}.79 \\
.49 \\
.93 \\
.63\end{array}$ & $\begin{array}{l}.79 \\
.43 \\
.93 \\
.69\end{array}$ & $\begin{array}{l}.80 \\
.48 \\
.88 \\
.63\end{array}$ & $\begin{array}{r}1434 \\
391 \\
207 \\
778\end{array}$ \\
\hline $\mathbf{A}$ & $\begin{array}{l}S_{2} I_{2} S_{2} \\
S_{1} I_{2} S_{2} \\
S_{2} I_{2} S_{1} \\
S_{1} I_{2} S_{1}\end{array}$ & $\begin{array}{l}.43 \\
.25 \\
.52 \\
.34\end{array}$ & $\begin{array}{l}.45 \\
.26 \\
.51 \\
.32\end{array}$ & $\begin{array}{l}.41 \\
.25 \\
.50 \\
.37\end{array}$ & $\begin{array}{r}281 \\
1125 \\
625 \\
177\end{array}$ & $\begin{array}{l}.56 \\
.20 \\
.74 \\
.38\end{array}$ & $\begin{array}{l}.57 \\
.19 \\
.73 \\
.33\end{array}$ & $\begin{array}{l}.50 \\
.17 \\
.68 \\
.39\end{array}$ & $\begin{array}{r}445 \\
1716 \\
579 \\
137\end{array}$ & $\begin{array}{l}.74 \\
.41 \\
.90 \\
.57\end{array}$ & $\begin{array}{l}.79 \\
.40 \\
.88 \\
.56\end{array}$ & $\begin{array}{l}.77 \\
.39 \\
.88 \\
.58\end{array}$ & $\begin{array}{r}420 \\
1576 \\
699 \\
194\end{array}$ & $\begin{array}{l}.79 \\
.49 \\
.93 \\
.63\end{array}$ & $\begin{array}{l}.83 \\
.49 \\
.90 \\
.63\end{array}$ & $\begin{array}{l}.73 \\
.44 \\
.92 \\
.69\end{array}$ & $\begin{array}{r}405 \\
1439 \\
702 \\
189\end{array}$ \\
\hline$x^{2} / d f(d$ & & 2.46 & 3.49 & & & 2.78 & 4.67 & & & 4.39 & 3.54 & & & 4.74 & 9.39 & & \\
\hline
\end{tabular}


Table 5

The Transition Matrix for the Memory State Models of Recognition for Random Sequences of Signal Events Extended to Inchude the Case in Which $O$ Has Information About Signal Probabilities. A prime on the parameter indicates that its value is subtracted from 1.0.

\begin{tabular}{cccccc} 
& & \multicolumn{4}{c}{ State on Trial n } \\
\cline { 3 - 5 } & & $\psi_{1}$ & $\psi_{2}$ & $\psi_{3}$ & $\psi_{4}$ \\
\hline \multirow{2}{*}{ State on } & $\psi_{1}$ & $\gamma \alpha$ & $\gamma^{\prime} \alpha$ & $\alpha^{\prime}(\Gamma+\epsilon)$ & $\alpha^{\prime}\left(\Gamma^{\prime}-\epsilon\right)$ \\
Trial n -1 & $\psi_{2}$ & $\gamma \alpha$ & $\gamma^{\prime} \alpha$ & $\alpha^{\prime}(\Gamma-\epsilon)$ & $\alpha^{\prime}\left(\Gamma^{\prime}+\epsilon\right)$ \\
& $\psi_{3}$ & $\gamma \alpha$ & $\gamma^{\prime} \alpha$ & $\alpha^{\prime}(\Gamma+\imath)$ & $\alpha^{\prime}\left(\Gamma^{\prime}-\imath\right)$ \\
& $\psi_{4}$ & $\gamma \alpha$ & $\gamma^{\prime} \alpha$ & $\alpha^{\prime}(\Gamma-\imath)$ & $\alpha^{\prime}\left(\Gamma^{\prime}+i\right)$ \\
\hline
\end{tabular}

$\alpha=.65, \Delta=1$, for $\gamma=.8, \delta_{1}=.02$, $\delta_{2}=-.73$, and for $\gamma=.2, \delta_{1}=.78$, $\delta_{2}=-.01$ ).

For the same data, the MS model requires but three parameters, $\alpha=.59$, $\epsilon=.06$, and $\iota=.06$

The predicted proportions for the first-order signal-response dependencies for the MS and MTR models are given in Table 6 with the observed proportions (Obs) and the number of observations ( $N$ ), which the authors of that paper kindly supplied. The chi-square deviations for the fits of the two models given at the bottom of Table 6 are significant $(p<.01)$; however, neither model is significantly better than the other.

\section{CONCLUSION}

The psychological nature of the stimulus and response effects described differs widely for the two models. The MTR model attributes sequential contrast to sensory differences between observed signal effects and the memory for previous observations. The MS model attributes these effects solely to response strategies adopted by the $O$, with the sensory effects invariant over manipulations of signal probabilities and sequential structure. It should be noted that for the MS model, in the

\section{Table 6}

Observed and Predicted Values of $\operatorname{Pr}\left(I_{2, n} / S_{i, n} I_{i, n-1} S_{i, n-1}\right)$ for the Feedback Condition from Tanner et al (1970)

Predicted

\begin{tabular}{rrrrrr}
$\operatorname{Pr}\left(S_{1}\right)$ & Seq. & MS & MTR & Obs. & \multicolumn{1}{c}{$N$} \\
\hline & $S_{2} I_{2} S_{2}$ & .94 & .94 & .95 & 5592 \\
& $S_{1} I_{2} S_{2}$ & .35 & .35 & .30 & 428 \\
& $S_{2} I_{2} S_{1}$ & .94 & .94 & .92 & 432 \\
.8 & $S_{1} I_{2} S_{1}$ & .35 & .43 & .62 & 83 \\
& $S_{2} I_{1} S_{2}$ & .89 & .83 & .88 & 346 \\
& $S_{1} I_{1} S_{2}$ & .30 & .26 & .31 & 33 \\
& $S_{2} I_{1} S_{1}$ & .89 & .92 & .89 & 951 \\
& $S_{1} I_{1} S_{1}$ & .30 & .28 & .19 & 55 \\
& $S_{2} I_{2} S_{2}$ & .70 & .72 & .75 & 245 \\
& $S_{1} I_{2} S_{2}$ & .11 & .08 & .08 & 973 \\
& $S_{2} I_{2} S_{1}$ & .70 & .71 & .54 & 103 \\
& $S_{1} I_{2} S_{1}$ & .11 & .16 & .10 & 396 \\
.2 & $S_{2} I_{1} S_{2}$ & .65 & .57 & .46 & 147 \\
& $S_{1} I_{1} S_{2}$ & .06 & .06 & .08 & 588 \\
$S_{2} I_{1} S_{1}$ & .65 & .62 & .63 & $\mathbf{9 1 7}$ \\
& $S_{1} I_{1} S_{1}$ & .06 & .05 & .06 & $\mathbf{5 8 5 5}$
\end{tabular}

$\chi^{2} /$ df (dev.) 11.4215 .62 case of Markov chains, changes in the apparent sensitivity to the two signals are accounted for by response bias only. For the MTR model, in the case of presentation probability variations, apparent bias changes are accounted for by changes in sensory-memory sensitivity. The interpretation of these changes depends on the model used and not on the apparent changes indicated by shifts on an ROC plot.

Both the MS and the MTR models do well with the random, Markov, and feedback data. The MS model does, however, capture the first-order effects with fewer parameters and correctly describes the nature of higher order effects. Further, the three-state threshold model with response determined by a first-order Markov process is mathematically more tractable and simpler than the process described by the MTR model. In any case, the MTR model must be reformulated in order to deal with the response strategies used by $O$ s in the Markov chain experiment. The memory to which the current signal effect is compared must be described as an adaptation-level point, subjectively between the recognition signals rather than as the memory of the preceding signal.

The MS model must be reformulated to deal with information which the $O$ has concerning signal probabilities. It provides that in the nonrecognition state, $O$ repeats his previous response at the subjective stimulus probability rates. This repetition rate can be modified by prior stimulus information when no feedback is given, or, when feedback is given, modified by the trial-to-trial information provided by the $\mathrm{E}$.

It is surprising that two theories so divergent in their underlying assumptions can capture the array of complex effects represented in this report. It is clear from these and previous experimental data that both models can predict the response pattern, assimilation or contrast, under a variety of signal presentation and feedback conditions.

Previous papers (Kinchla \& Smyzer, 1967; Sandusky \& Ahumada, 1970; Parducci \& Sandusky, 1970) have examined the psychophysical conditions in which probability and sequential contrast or assimilation can be expected. In the absence of an immediate comparative sensory basis for judgment, as in detection with the noise gated on only during the observation interval, or in recognition without a background reference, contrast predominates. Where there is a comparative basis, as in detection with continuous noise or a continuous reference in recognition, or when trial-by-trial feedback is provided, signal probability learning effects predominate and assimilation is observed. It is probable that both judgmental-contrast and learning-assimilation effects are present to some degree in all psychophysical tasks.

\section{REFERENCES}

ANDERSON, N. An analysis of sequential dependencies. In $R$. R. Bush and $W$. $K$. Estes (Eds), Studies in mathematical learning theory. Stanford: Stanford University Press, 1959

ATKINSON, R. C., BOWER, G. H., \& CROTHERS, E. D. An introduction to mathematical learning theory. New York: Wiley, 1965.

ATKINSON, R. C., CARTERETTE, E. C., \& KINCHLA, R. A. Sequential phenomena in psychophysical judgments: A theoretical analysis. I.R.E. Transactions on Information Theory, 1962, IT-8S, 155-162.

BUSH, B. R., \& MOSTELLER, F Stochastic models of learning. New York: Wiley, 1955.

FRIEDMAN, M. P., CARTERETTE, E. C., NAKATANI, L. \& AHUMADA. A Feedback and frequency variables in signal detection. Perception \& Psychophysics, 1968, 3, 5-10

HALLER, $R$. W. The role of information feedback in signal recognition. Unpublished doctoral dissertation. Stanford University, 1969.

HELSON, H. Adaptation level theory. New York: Harper \& Row, 1965.

KINCHLA, R. A., \& SMYZER, F, A diffusion model of perceptual memory. Perception \& Psychophysics, 1967, 2, 219-229.

PARDUCCI. A. Sequential effects in judgment. Psychological Bulletin, 1964 61, 16-167.

PARDUCCI, A., \& SANDUSKY, A. Distribution and sequence effects in judgment. Journal of Experimental Psychology, 1965, 69, 450-459.

PARDUCCI, A., \& SANDUSKY, A. Limits on the applicability of signal detection theory. Perception \& Psychophysics. $1970,7,63-64$.

SANDUSKY, A. Two-choice recognition of stimuli in Markovian sequences: A test of two models. Unpublished doctoral dissertation, University of Califormia, Los Angeles, 1966

SANDUSKY, A., \& AHUMADA, A Contrast in detection with gated noise. Joumal of the Acosutical Society of America. 1971, in press.

SUPPES, P., \& ATKINSON, R. C. Markov learning models for multiperson interactions. Stanford: Stanford University Press, 1960.

TANNER, T. A., HALLER, R. W., \& ATKINSON, R. C. Signal recognition as influenced by presentation schedules. Perception \& Psychophysics, 1967, 2 349358. 
TANNER, T. A., JR., RAUK, J. A., \& ATKINSON, R. C. Signal recognition as influenced by information feedback. Journal of Mathematical Psychology, $1970,7,259-274$.
THURSTONE, L. I. A law of comparative judgment. Psychological Review, 1927 , $34,273-286$.

TORGERSON, W. S. Theory and methods of scaling. New York: Wiley, 1958.

(Accepted for publicalion April 23, 1971.) 